\title{
Age-Related Hearing Loss, Late-Life Depression, and Risk for Incident Dementia in Older Adults
}

\author{
Katharine K. Brewster, MD, ${ }^{1, *, 0}$ Mei-Chen Hu, PhD, ${ }^{1}$ Sigal Zilcha-Mano, PhD, ${ }^{1,2}$ \\ Alexandra Stein, BA, ${ }^{1}$ Patrick J. Brown, PhD, ${ }^{1}$ Melanie M. Wall, PhD, ${ }^{1}$ Steven P. Roose, \\ MD, ${ }^{1}$ Justin S. Golub, MD, MS, ${ }^{3}$ and Bret R. Rutherford, MD ${ }^{1}$
}

${ }^{1}$ New York State Psychiatric Institute, Columbia University College of Physicians and Surgeons, New York. ${ }^{2}$ Department of Psychiatry, University of Haifa, Israel. ${ }^{3}$ Columbia University Department of Otolaryngology-Head and Neck Surgery, Columbia University College of Physicians and Surgeons, New York.

*Address correspondence to: Katharine K. Brewster, MD, New York State Psychiatric Institute, Columbia University College of Physicians and Surgeons, 1051 Riverside Drive, Box 92, New York, NY 10032. E-mail: Katharine.Brewster@nyspi.columbia.edu

Received: April 22, 2020; Editorial Decision Date: September 16, 2020

Decision Editor: Anne B. Newman, MD, MPH, FGSA

\begin{abstract}
Background: Hearing loss (HL), late-life depression, and dementia are 3 prevalent and disabling conditions in older adults, but the interrelationships between these disorders remain poorly understood.

Methods: $N=8529$ participants $\geq 60$ years who were free of cognitive impairment at baseline were analyzed from National Alzheimer's Coordinating Center Uniform Data Set. Participants had either No HL, Untreated HL, or Treated HL. Primary outcomes included depression (15-item Geriatric Depression Scale $\geq 5$ ) and conversion to dementia. A longitudinal logistic model was fit to examine the association between HL and changes in depressive symptoms across time. Two Cox proportional hazards models were used to examine HL and the development of dementia: Model A included only baseline variables and Model B included time-varying depression to evaluate for the direct effect of changes in depression on dementia over time.

Results: Treated HL (vs no HL) had increased risk for depression (odds ratio $[\mathrm{OR}]=1.26,95 \%$ confidence interval $[\mathrm{CI}]=1.04-1.54, p=.02$ ) and conversion to dementia (hazard ratio $[\mathrm{HR}]=1.29,95 \% \mathrm{CI}=1.03-1.62, p=.03$ ). Baseline depression was a strong independent predictor of conversion to dementia $(\mathrm{HR}=2.32,95 \% \mathrm{CI}=1.77-3.05, p<.0001)$. Development/persistence of depression over time was also associated with dementia $(\mathrm{HR}=1.89,95 \% \mathrm{CI}=1.47-2.42, p<.0001)$, but only accounted for $6 \%$ of the direct hearing-dementia relationship (Model A $\log H \mathrm{R}=0.26[S E=0.12]$ to Model B $\log \mathrm{HR}=0.24[S E=0.12]$ ) suggesting no significant mediation effect of depression.

Conclusions: Both HL and depression are independent risk factors for eventual conversion to dementia. Further understanding the mechanisms linking these later-life disorders may identify targets for early interventions to alter the clinical trajectories of at-risk individuals.
\end{abstract}

Keywords: Cognitive impairment, Dementia, Hearing Loss, Late-life depression

Age-related hearing loss (ARHL) is the third most common health condition affecting older adults after heart disease and arthritis (1). Its prevalence rises steeply with age, from $3 \%$ among adults aged $20-29$ years to $49 \%$ of adults aged $60-69$ and over $80 \%$ in individuals 85 years and older $(2,3)$. An estimated 38 million Americans now suffer from untreated ARHL (4). While historically considered a benign effect of aging or exclusively a quality of life issue, ARHL has been associated with negative health outcomes including social isolation, frailty, and falls (5). Age-related hearing loss is now the fifth leading cause of years lived with disability worldwide, and this rank is expected to rise as the population continues to skew older (6).

Growing evidence also links ARHL to adverse neuropsychiatric outcomes such as dementia and late-life depression. Secondary analyses of epidemiologic data sets $(7,8)$, reviews (9), meta-analyses (10), and a National Institute on Aging workshop on the topic (11), all have linked hearing loss to cognitive decline and dementia. Studies by our group and others reported both 
cross-sectional and longitudinal associations between hearing loss and increased depressive symptoms as well as syndromal depression in older adults $(12,13)$. Though the specific pathways involved remain to be specified, it is hypothesized that ARHL increases risk for late-life depression and dementia via behavioral mechanisms such as decreased social engagement in addition to deleterious neuroplastic changes secondary to deafferentation (14).

Like ARHL, depression itself is a risk factor for cognitive decline and dementia (15). Experiencing depression within the past 10 years increases the risk of dementia 4- to 6-fold for older adults $(16,17)$, and multiple reports suggest that a single depressive episode at any point in life is a risk factor for dementia $(18,19)$. A chronic illness or recurrent episodes are common in late-life depression, and individuals with persistent depressive symptoms, especially when combined with cognitive deficits, place older adults at even higher risk for dementia $(20,21)$. It has been hypothesized that late-life depression, particularly when it is persistent and treatment resistant, is associated with increased levels of pro-inflammatory markers (22), cerebrovascular lesions (23), and cognitive deficits (24) that may lie in a causal chain leading to dementia or else may speed conversion to dementia by decreasing brain reserve. Some estimate that successful interventions targeting late-life depression may reduce the prevalence of Alzheimer's disease by up to $15 \%$ in North America (25), making depression in older adults the second most promising target for dementia prevention after physical inactivity.

While the studies reviewed above examine pairwise links between hearing loss, depression, and dementia, an understanding of the interrelationships among these 3 prevalent and disabling conditions of later life remains elusive. Elucidating the degree to which these disorders share pathophysiologic processes may facilitate identification of targets for prevention and treatment. In this study, we utilized the National Alzheimer's Coordinating Center's (NACC) longitudinal database of older adults, which incorporates comprehensive neuropsychiatric assessments and rigorous classification of cognitive status. Our primary aims were to evaluate the neuropsychiatric effects of hearing loss in a cohort of participants $\geq 60$ years who were free of mild cognitive impairment (MCI) or dementia at baseline. We hypothesized that (i) hearing loss would predict depression as well as conversion to dementia over follow-up, and (ii) the development of depression would partially mediate the hearing-dementia association.

\section{Method}

\section{Study Population}

From 2005 to the present, NACC has collected the Uniform Data Set (UDS) on participants from approximately 30 past and present U.S. Alzheimer's Disease Research Centers. Participants with clinical diagnoses of normal cognition, MCI, and dementia of various etiologies were enrolled and followed approximately annually. To compose our analytic sample, we identified a cohort among NACC participants $\geq 60$ years who were free of dementia or MCI at baseline, presented for at least 2 follow-up visits or 1 follow-up visit followed by death within a year, and had baseline hearing and depression assessments. Baseline was defined as either the initial UDS visit for participants $\geq 60$ years or the first follow-up visit after the participant reached 60 years.

\section{Assessments}

Hearing loss and hearing aid use were assessed at baseline and at each follow-up visit by a clinical interview and physical examination. The clinician responded to items querying (1) "Without a hearing $\operatorname{aid}(\mathrm{s})$, is the subject's hearing functionally normal?” $(0=$ Normal Hearing, 1 = Impaired Hearing), (2) "Does the subject wear a hearing $\operatorname{aid}(\mathrm{s})$ ?" $(0=$ No Aid, $1=$ Hearing Aid $)$, and (3) "If the subject usually wears a hearing aid(s), is the subject's hearing functionally normal with a hearing aid(s)?” ( $0=$ Normal Hearing with Aid, 1 = Impaired Hearing with Aid). Utilizing clinicians' responses to these items, we classified participants into categories of "No Hearing Loss," "Untreated Hearing Loss," and "Treated Hearing Loss" at baseline. The No Hearing Loss category comprised individuals with normal hearing who did not use a hearing aid (ie, 0 on Question 1, 0 on Question 2). Participants classified as Untreated Hearing Loss had hearing loss and either no hearing aid or else continued hearing impairment despite hearing aid use ( 1 on Question 1, 0 on Question 2, or 1 on Question 2 combined with 1 on Question 3). Finally, the Treated Hearing Loss category included participants who wore hearing aids with consequent perceived functionally normal hearing (ie, 1 on Question 2, 0 on Question 3).

Depressive symptoms were assessed using the 15 -item Geriatric Depression Scale (GDS) (26) at the initial UDS and at each follow-up visit. The 15 -item GDS uses a yes/no answer format and scores range from 0 to 15 . A cutoff of 5 or greater on this short-form 15-item GDS has shown to be highly sensitive to the identification of major depression (27). Cognitive status was evaluated at baseline and at each follow-up visit through a history and physical, behavioral assessments (Clinical Dementia Rating (28), Neuropsychiatric Inventory Questionnaire (29)), functional assessments (Functional Assessment Questionnaire (30)), and a comprehensive neuropsychological battery (31). Participants were classified as having normal cognition, MCI, or dementia. Criteria for classification of MCI and dementia were made across all Alzheimer's Disease Centers using guidelines set forth by the Alzheimer's Disease Centers Clinical Task Force (32). We defined time to dementia as the number of months from baseline until the first visit with a diagnosis of dementia. To ensure reliability of the diagnosis, participants were only deemed to have dementia if either the diagnosis occurred in 2 consecutive visits or if the diagnosis was followed by a death within 1 year. A dementia diagnosis followed by a classification of MCI or only one dementia diagnosis at the last visit was not considered dementia. Blood samples were collected to determine APOE $\varepsilon 4$ status, and individuals were classified as having lowest risk (no $\varepsilon 4$ alleles), intermediate risk (1 14 allele), and highest risk ( 2 \&4 alleles).

\section{Statistical Analysis}

Descriptive summaries of hearing loss categories by demographics, baseline depression, and average length of follow-up were calculated and tested for differences using multinomial regression. To examine the association between hearing loss and changes in depressive symptoms across time, a longitudinal logistic model was fit with generalized estimating equations. The longitudinal outcome was a dichotomous indicator at each observed follow-up visit of whether the participant had or did not have GDS $\geq 5$ at that visit. Predictors included baseline hearing category, baseline depression (ie, dichotomous GDS $\geq 5$ yes/no), and time since baseline (continuous) corresponding to the follow-up visit. All analyses were estimated after controlling for age, sex, ethnicity, education (in years of schooling, range 0-29), and APOE status. 
To account for repeated depression measures within person, a compound symmetry working correlation matrix was used within the generalized estimating equations. Odds ratios (ORs) and $95 \%$ confidence intervals (CIs) provide the estimated strength of association between each predictor and the odds of depression over time. Additional models tested for interactions between baseline hearing loss and baseline depression and demographics to identify if any group had a stronger or weaker association between hearing and longitudinal depression.

To examine the association between hearing loss, depression, and development of dementia, we used Cox proportional hazards models with time from baseline until time of the dementia diagnosis as the primary outcome. Censoring time for those not developing dementia was taken to be death or the last observed visit with no dementia diagnosis. We fit 2 models. The first (Model A) included only baseline hearing status, baseline depression, and control variables age, sex, ethnicity, education, and APOE status. The second (Model B) included baseline hearing status and both baseline depression and a time-varying depression variable to evaluate the direct effect of changing depressive symptom severity on dementia over time. To avoid potential conflation of reverse causality due to diagnoses of both depression and dementia occurring at the same visit, we only counted a new depression diagnosis if it was observed prior to the visit of the initial dementia diagnosis. For participants without dementia, the depression was counted during follow-up regardless of when it occurred.

Finally, we tested a mediation hypothesis that hearing loss increases risk for conversion to dementia through its effect on depression. The models described above (longitudinal model for hearing to depression and Cox model for hearing and depression to dementia) make up the components of the mediation test. We obtained the indirect effect and associated CI following modern methods for estimation of causal mediation effects (33).

\section{Results}

\section{Sample Characteristics}

As shown in Figure 1, $N=36799$ participants were available for analysis of the UDS visits conducted between September 2005 and December 2017. Of these individuals, $N=14491$ were free of dementia or MCI at baseline, $N=9059$ participants had at least 2 follow-up visits, or else 1 follow-up visit followed by death within a year. $N=530$ participants were excluded for having missing hearing or depression data, resulting in a final analytic sample of $N=8529$ participants.

As shown in Table 1, of the 8529 included participants, 1102 $(12.9 \%)$ were categorized as having Treated Hearing Loss and 949 $(11.1 \%)$ participants as having Untreated Hearing Loss. As expected, the 2 groups with hearing loss were older $\left(X^{2}(6)=443.17\right.$, $p<.0001)$ and more likely to be male $\left(X^{2}(2)=84.00, p<.0001\right)$, especially for those with Treated Hearing Loss. Those with Treated Hearing Loss were less likely to be Hispanic, or non-Hispanic African American than non-Hispanic White $\left(X^{2}(8)=80.75, p\right.$ $<.0001)$, and more likely to have a higher number of years of schooling $\left(X^{2}(2)=9.57, p=.0084\right)$. Those with Untreated Hearing Loss were less likely to have an intermediate or high risk APOE genotype $\left(X^{2}(6)=14.99, p=.0203\right)$. There was no significant difference in depressive symptoms at baseline between the hearing groups. The average length of follow-up in the sample was 67 months (range 9-144 months).

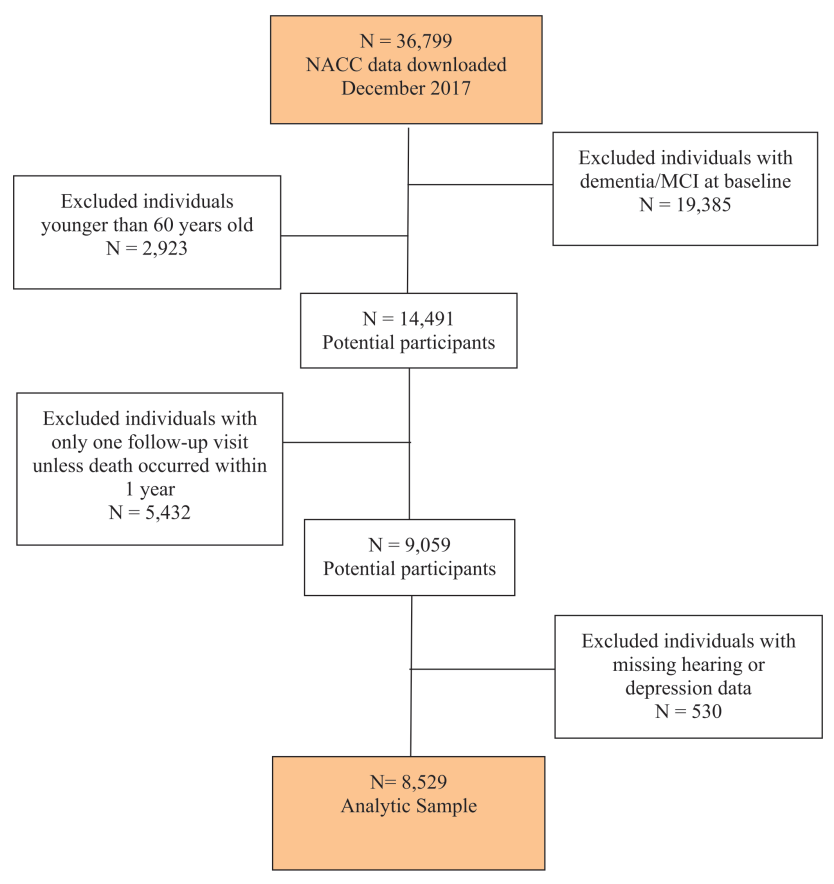

Figure 1. Flow diagram of included participants. Full color version is available within the online issue.

\section{Hearing Loss and Risk of Depression Over Time}

Relative to those with No Hearing Loss, individuals with Treated Hearing Loss had higher odds of depression ( 15 -item GDS $\geq 5$ ) across follow-up $(\mathrm{OR}=1.26,95 \% \mathrm{CI}=1.04-1.54, p=.02)$ after controlling for baseline depression and demographics (Table 2). Those with Untreated Hearing Loss did not show significantly higher odds of depression. Both older and female individuals showed higher odds of depression, while non-Hispanic African Americans and number of years of schooling demonstrated lower odds. There were no differential effects of hearing loss on depression by baseline depression, age, and gender groups.

\section{Hearing Loss, Depression, and Incident Dementia}

In the Cox model considering only baseline variables as predictors (Model A), individuals with Treated Hearing Loss were at higher risk for conversion to dementia compared to those with No Hearing Loss (hazard ratio $[\mathrm{HR}]=1.29,95 \% \mathrm{CI}=1.03-1.62, p=.03)$. Baseline depression was also found be a strong predictor of conversion to dementia $(\mathrm{HR}=2.32,95 \% \mathrm{CI}=1.77-3.05, p<.0001)$. Older age, male sex, White race (as compared to African American), and having an intermediate or high risk $A P O E$ genotype all increased risk of dementia while number of years of schooling decreased the risk (Table 3). When time-varying depression was considered (Model B), it was found to further increase the risk of dementia $(\mathrm{HR}=1.89$, $95 \% \mathrm{CI}=1.47-2.42, p<.0001)$ above and beyond the effect of baseline depression and Treated Hearing Loss. When depression occurring during follow-up was considered (ie, in Model B), the effect of Treated Hearing Loss on dementia remained significant $(\mathrm{HR}=1.27,95 \% \mathrm{CI}=1.01-1.59, p=.04)$. Between Model A and Model B, the effect of Treated Hearing Loss on dementia was only reduced by $6 \%$ (from $\log \mathrm{HR}=0.26[S E=0.12]$ in Model $\mathrm{A}$ to $\operatorname{logHR}=0.24[S E=0.12]$ in Model B), suggesting that depression was not a significant mediator of the hearing-dementia link. 
Table 1. Baseline Demographics of the Analytic Sample $(N=8529)$ and Characteristics of the Hearing Categories

\begin{tabular}{|c|c|c|c|c|c|}
\hline & $\begin{array}{l}\text { No Hearing Loss } \\
(N=6478)\end{array}$ & $\begin{array}{l}\text { Untreated } \\
\text { Hearing Loss } \\
(N=949)\end{array}$ & $\begin{array}{l}\text { Treated Hearing } \\
\text { Loss }(N=1102)\end{array}$ & $\begin{array}{l}\text { Total } \\
(N=8529)\end{array}$ & \multirow[b]{2}{*}{$X^{2}, p$ Value } \\
\hline & \multicolumn{4}{|c|}{ Mean $\pm S D$ or $N(\%)$} & \\
\hline Age & $72.8 \pm 7.5$ & $75.2 \pm 8.0$ & $78.9 \pm 7.8$ & $73.9 \pm 7.9$ & \multirow{5}{*}{$\begin{array}{l}X^{2}(6)=443.17, \\
p<.0001\end{array}$} \\
\hline $60-69$ & $2403(37.1)$ & $265(27.9)$ & 155 (14.1) & $2823(33.1)$ & \\
\hline $70-79$ & $2783(43.0)$ & $394(41.5)$ & $388(35.2)$ & $3565(41.8)$ & \\
\hline $80-89$ & $1161(17.92)$ & $253(26.7)$ & $466(42.3)$ & $1880(22.0)$ & \\
\hline $90+$ & $131(2.0)$ & $37(3.9)$ & $93(8.4)$ & $260(3.06)$ & \\
\hline \multicolumn{5}{|l|}{ Sex } & \multirow{3}{*}{$\begin{array}{l}X^{2}(2)=84.00 \\
p<.0001\end{array}$} \\
\hline Males & $2159(33.3)$ & $400(42.2)$ & $557(50.5)$ & $3117(36.6)$ & \\
\hline Females & 4319 (66.7) & $549(57.85)$ & $545(49.5)$ & $5412(63.45)$ & \\
\hline \multicolumn{5}{|l|}{ Race/ethnicity } & \multirow{6}{*}{$\begin{array}{l}X^{2}(8)=80.75, \\
p<.0001\end{array}$} \\
\hline Non-Hispanic White & $4863(75.1)$ & $712(75.0)$ & $1,008(91.5)$ & $6583(77.2)$ & \\
\hline Non-Hispanic African American & $1039(16.0)$ & $145(15.3)$ & $44(4.0)$ & $1228(14.4)$ & \\
\hline Hispanic & $392(6.1)$ & $69(7.3)$ & $32(2.9)$ & $493(5.8)$ & \\
\hline Non-Hispanic Asian & $134(2.1)$ & $19(2.0)$ & $14(1.3)$ & $167(2.0)$ & \\
\hline \multicolumn{5}{|l|}{ Native Hawaiian, missing) } & \\
\hline Years of schooling* & $15.5(3.1)$ & $15.5(3.4)$ & $16.0(2.9)$ & $15.6(3.1)$ & $\begin{array}{l}X^{2}(2)=9.57 \\
p=.0084\end{array}$ \\
\hline \multicolumn{5}{|l|}{ APOE genotype } & \multirow{5}{*}{$\begin{array}{l}X^{2}(6)=14.99 \\
p=.0203\end{array}$} \\
\hline Lowest risk (no $\varepsilon 4$ alleles) & $4125(63.7)$ & $657(69.2)$ & $747(67.8)$ & $5529(64.8)$ & \\
\hline Intermediate ( $1 \varepsilon 4$ allele) & $1682(26.0)$ & $193(20.3)$ & $260(23.6)$ & $2135(25.0)$ & \\
\hline High risk ( 2 \& 4 alleles) & $148(2.3)$ & $13(1.4)$ & $20(1.8)$ & $181(2.1)$ & \\
\hline Missing & $523(8.1)$ & $86(9.1)$ & $75(6.8)$ & $684(8.0)$ & \\
\hline Duration follow-up (months) & $67.5 \pm 33.5$ & $66.9 \pm 34.3$ & $64.2 \pm 32.9$ & $67.0 \pm 33.5$ & \multirow{6}{*}{$\begin{array}{l}X^{2}(2)=0.76 \\
p=.6839 \\
X^{2}(2)=0.95 \\
p=.6217\end{array}$} \\
\hline Depression $^{\dagger}$ at baseline & $476(7.3)$ & $72(7.6)$ & $65(5.9)$ & $613(7.2)$ & \\
\hline $\mathrm{GDS}^{\dagger}$ score at baseline & $1.37 \pm 2.07$ & $1.42 \pm 2.10$ & $1.33 \pm 1.86$ & $1.37 \pm 2.05$ & \\
\hline Depression $^{\dagger}$ during follow-up ${ }^{\ddagger}$ & $949(14.7)$ & $160(16.9)$ & $193(17.5)$ & $1302(15.3)$ & \\
\hline Mean time to dementia (months) & $66.9 \pm 33.5$ & $66.6 \pm 34.2$ & $63.1 \pm 32.9$ & $65.4 \pm 33.6$ & \\
\hline Proportion converting to dementia & $335(5.2)$ & $53(5.6)$ & $110(10.0)$ & $498(5.8)$ & \\
\hline
\end{tabular}

Notes: $S D=$ standard deviation.

*Years of schooling measured as a continuous variable (range 0-29).

${ }^{\dagger}$ Depression = 15 -item Geriatric Depression Scale (GDS) $\geq 5$.

‡Depression during follow-up that occurred prior to dementia diagnosis. One hundred fifty-eight participants had missing data on depression during follow-up.

\section{Discussion}

This analysis of 8529 older adults contributing to the NACC database found important interrelationships between hearing loss, latelife depression, and dementia, which are 3 of the most prevalent and consequential disorders of later life. We found that Treated Hearing Loss predicted both depression and conversion to dementia over an average follow-up period of 5.6 years. Likewise, our analyses reconfirmed previous findings that depression is a significant predictor of conversion to dementia. The associations between hearing loss, depression, and dementia were not only statistically significant but were also clinically meaningful. The highest risk hearing loss category increased the risks of both depression and dementia across time by over $25 \%$, and participants who began the study with significant depression were over 2.3 times more likely to convert to dementia compared to those without depression.

The finding that Treated rather than Untreated Hearing Loss was associated with increased risk for depression and dementia was surprising, as we expected treatment of hearing loss would be protective against adverse neuropsychiatric consequences. Prior studies have not disambiguated the effects of treated and untreated hearing loss on the development of depression and dementia. As this finding may represent a type I error, it must be replicated by future analyses of independent data sets. It also is possible, though we consider it unlikely, that hearing aid treatment has harmful effects on mood and cognition. However, the most likely interpretation of these findings is that hearing aid usage is associated with longer duration, more severe hearing loss (34), which itself increases risk for depression and dementia. Furthermore, hearing aid compliance rates are usually low and inaccurately reported (35), and we suspect that most individuals who reported to clinicians that hearing aids normalized their hearing were not in fact receiving adequate hearing treatment.

The findings reported here with respect to hearing loss partially diverge from 2 previous NACC publications, which did not report significant associations between hearing loss and incident Alzheimer's disease (36) or MCI (37). The primary focus of these reports was to identify specific characteristics of depressed older adults that predict cognitive decline, and toward that end the contribution of several medical conditions, health behaviors, and physical examination findings were examined. These studies examined smaller sample sizes (1965 and 2655 participants, respectively, as compared to 8529 in this analysis) and required the presence of baseline depressive illness, which may account for the difference in 
Table 2. Relationships Between Hearing Loss, Sociodemographic Characteristics, and Depression During Follow-up

\begin{tabular}{|c|c|c|c|c|}
\hline & \multicolumn{4}{|c|}{ Depression During Follow-up } \\
\hline & Odds Ratio & $95 \% \mathrm{CI}$ & $Z$ & $p$ Value \\
\hline \multicolumn{5}{|l|}{ Baseline hearing status } \\
\hline No Hearing Loss (ref.) & 1.00 & & & \\
\hline Treated Hearing Loss & 1.26 & $1.04-1.54$ & 2.34 & .02 \\
\hline Untreated Hearing Loss & 1.10 & $0.90-1.35$ & 0.94 & .35 \\
\hline Depression" at baseline & 14.47 & $12.37-16.93$ & 33.39 & $<.0001$ \\
\hline \multicolumn{5}{|l|}{ Age } \\
\hline 60-69 (ref.) & 1.00 & & & \\
\hline $70-79$ & 1.19 & $1.02-1.39$ & 2.17 & .03 \\
\hline $80-89$ & 1.58 & $1.32-1.89$ & 4.91 & $<.0001$ \\
\hline$\geq 90$ & 2.58 & $1.87-3.56$ & 5.78 & $<.0001$ \\
\hline Female & 1.15 & $1.00-1.32$ & 2.03 & .04 \\
\hline \multicolumn{5}{|l|}{ Race/ethnicity } \\
\hline Non-Hispanic White (ref.) & 1.00 & & & \\
\hline Non-Hispanic African American & 0.74 & $0.60-0.91$ & -2.91 & .0036 \\
\hline Hispanic & 1.27 & $0.97-1.66$ & 1.77 & .08 \\
\hline Non-Hispanic Asian & 1.15 & $0.75-1.78$ & 0.64 & .53 \\
\hline Other & 0.94 & $0.51-1.75$ & -0.18 & .85 \\
\hline Years of schooling ${ }^{\dagger}$ & 0.96 & $0.94-0.99$ & -3.22 & .0013 \\
\hline \multicolumn{5}{|l|}{ APOE genotype } \\
\hline Lowest risk (no ع4 alleles) (ref.) & 1.00 & & & \\
\hline Intermediate ( $1 \varepsilon 4$ allele) & 1.00 & $0.85-1.17$ & -0.03 & .98 \\
\hline High risk ( $2 \varepsilon 4$ alleles) & 0.96 & $0.64-1.43$ & -0.19 & .85 \\
\hline Time from baseline to follow-up visit (months) & 1.0027 & $1.0011-1.0043$ & 3.24 & .0012 \\
\hline
\end{tabular}

Notes: $\mathrm{CI}=$ confidence interval.

*Depression = 15-item Geriatric Depression Scale (GDS) $\geq 5$.

tYears of schooling measured as a continuous variable (range 0-29).

the findings for hearing loss. In sensitivity analyses classifying participants as having Hearing Loss (combined Treated and Untreated) and No Hearing Loss, we found that those with Hearing Loss (vs No Hearing Loss) had significantly higher odds of depression across time $(\mathrm{OR}=1.19,95 \% \mathrm{CI}=1.02-1.38, p=.03)$ after controlling for baseline depression and demographics. However, in the Cox model considering only baseline variables as predictors (Model A), we found that individuals with Hearing Loss (vs No Hearing Loss) were not at higher risk for conversion to dementia $(\mathrm{HR}=1.18$, $95 \%$ CI $=0.97-1.43, p=.09$ ), results consistent with previous NACC analyses. While the classification of hearing loss into Treated and Untreated categories adds complexity to our data interpretation, our impression is that the distinction between Treated and Untreated Hearing Loss in the NACC UDS is an important one and may recognize severity and duration of hearing loss as risk factors for dementia. Moreover, our analyses also incorporated a model with time-varying depression, a method that may have allowed us to more accurately evaluate the relationships between hearing, depression, and cognition.

To our knowledge, this is the first study to perform a mediation analysis of hearing loss, late-life depression, and dementia. Consistent with past reports $(38,39)$, we found that persistent depression, as opposed to depression only present at baseline, was associated with the highest HR for conversion to dementia. We hypothesized that hearing loss may initiate a deleterious cascade of events leading away from healthy aging trajectories toward increased social and behavioral disengagement (40), executive dysfunction and decreased cognitive reserve (41), and secondary atrophy of brain regions connected to auditory circuits (42) and may explain the mechanistic links between hearing loss, depression, and cognitive decline. However, in our full model containing hearing loss and time-varying depression, we found that depression was only responsible for $6 \%$ of the hearing-dementia relationship, which did not support our hypothesis that depression mediates the link between hearing and dementia.

As our analyses did not support a significant mediation effect of depression on the hearing-dementia relationship, one explanation is that both depression and ARHL are prevalent but independent processes associated with age that increase risk for dementia. Another possibility is that clinician diagnosed hearing loss, rather than measurement of pure-tone hearing thresholds, may have introduced bias into our data collection. The NACC protocols for clinician examinations are not completely standardized and allow some variability between ADRC sites. While some Alzheimer's Disease Research Center (ARDC) clinicians may diagnose hearing loss by reviewing an audiometric evaluation if available, others rely on the participant's report of hearing loss. While self-reported hearing loss has demonstrated high sensitivity and specificity for hearing loss comparable to pure-tone audiometry in some reports $(43,44)$, it is possible that a participant's cognitive or depression status may have affected the examiner's perception of hearing function. Additionally, due to the chronicity of these processes as well the assessment tools available in the NACC UDS, it was difficult to disentangle the time of onset of both hearing loss and depression, which may have contributed to our inability to identify a mediation effect.

However, the above results suggest that screening for ARHL and depression in older adults and prompt institution of standard of care treatments for these conditions (ie, appropriately fitted and consistently worn hearing aids as well as pharmacologic or 
Table 3. Relationships Between Baseline Hearing Loss, Depression, Sociodemographic Characteristics, and Conversion to Dementia*

\begin{tabular}{|c|c|c|c|c|c|c|c|c|}
\hline & \multicolumn{4}{|l|}{ Model A } & \multicolumn{4}{|c|}{ Model B (time-varying depression) } \\
\hline & Hazard Ratio & $95 \% \mathrm{CI}$ & Chi-Square & $p$ Value & Hazard Ratio & $95 \% \mathrm{CI}$ & Chi-Square & $p$ Value \\
\hline \multicolumn{9}{|l|}{ Hearing Loss at baseline } \\
\hline No Hearing Loss (ref.) & 1.00 & & & & 1.00 & & & \\
\hline Treated Hearing Loss & 1.29 & $1.03-1.62$ & 4.86 & .03 & 1.27 & $1.01-1.59$ & 4.15 & .04 \\
\hline Untreated Hearing Loss & 1.02 & $0.76-1.36$ & 0.01 & .91 & 1.00 & $0.75-1.34$ & 0.00 & .99 \\
\hline Depression $^{\dagger}$ at baseline & 2.32 & $1.77-3.05$ & 36.38 & $<.0001$ & 1.65 & $1.21-2.23$ & 10.25 & .0014 \\
\hline Depression $^{\dagger}$ during follow-up & & & & & 1.89 & $1.47-2.42$ & 24.54 & $<.0001$ \\
\hline \multicolumn{9}{|l|}{ Age } \\
\hline 60-69 (ref.) & 1.00 & & & & 1.00 & & & \\
\hline $70-79$ & 2.79 & $2.08-3.74$ & 47.00 & $<.0001$ & 2.71 & $2.02-3.64$ & 44.40 & $<.0001$ \\
\hline $80-89$ & 6.46 & $4.80-8.71$ & 150.35 & $<.0001$ & 6.03 & $4.47-8.13$ & 138.51 & $<.0001$ \\
\hline$\geq 90$ & 15.16 & $10.12-22.70$ & 137.87 & $<.0001$ & 12.31 & $8.20-18.47$ & 147.02 & $<.0001$ \\
\hline Female & 0.75 & $0.62-0.90$ & 9.45 & .0021 & 0.76 & $0.63-0.91$ & 8.86 & .01 \\
\hline \multicolumn{9}{|l|}{ Race } \\
\hline Non-Hispanic White (ref.) & 1.00 & & & & 1.00 & & & \\
\hline Non-Hispanic African American & 0.63 & $0.46-0.86$ & 8.26 & .004 & 0.65 & $0.48-0.89$ & 7.09 & .0078 \\
\hline Hispanic & 1.12 & $0.75-1.65$ & 0.29 & .59 & 1.08 & $0.72-1.60$ & 0.13 & .72 \\
\hline Non-Hispanic Asian & 0.79 & $0.35-1.76$ & 0.34 & .56 & 0.75 & $0.33-1.68$ & 0.50 & .48 \\
\hline Other & 0.99 & $0.25-3.96$ & 0.00 & .98 & 0.88 & $0.22-3.54$ & 0.03 & .86 \\
\hline Years of schooling ${ }^{\ddagger}$ & 0.95 & $0.92-0.98$ & 10.12 & .0015 & 0.95 & $0.92-0.98$ & 9.81 & .0017 \\
\hline \multicolumn{9}{|l|}{ APOE genotype } \\
\hline Lowest risk (no $\varepsilon 4$ alleles) (ref.) & 1.00 & & & & & & & \\
\hline Intermediate ( $1 \varepsilon 4$ allele) & 2.15 & $1.78-2.61$ & 61.44 & $<.0001$ & 2.14 & $1.76-2.59$ & 59.93 & $<.0001$ \\
\hline High risk ( $2 \varepsilon 4$ alleles) & 3.89 & $2.48-6.10$ & 34.89 & $<.0001$ & 4.02 & $2.56-6.33$ & 36.31 & $<.0001$ \\
\hline
\end{tabular}

Notes: $\mathrm{CI}=$ confidence interval.

"Hazard ratios indicate the rate of conversion to dementia in the hearing categories and relevant covariates compared to the reference group for all included participants $(N=8529)$. The following interaction tests were performed: hearing * baseline depression $(p=.4170)$, hearing * time-varying depression $(p=.2284)$, hearing * age $(p=.7379)$, hearing * $\operatorname{sex}(p=.6694)$, and hearing * race/ethnicity $(p=.6825)$.

${ }^{\dagger}$ Depression $=15$-item Geriatric Depression Scale $(\mathrm{GDS}) \geq 5$.

‡Years of schooling measured as a continuous variable (range 0-29).

psychotherapeutic treatment of depression) is indicated. Given the links between ARHL and later-life neuropsychiatric disorders, the evaluation of an older patient presenting with subjective memory problems or depressive symptoms may be an important opportunity for clinicians to diagnose hearing loss. Clinicians can screen patients for hearing loss, refer patients for audiometric testing, and educate patients about the important health implications of these interrelated conditions and help patients overcome both logistical and psychological (ie, stigma) obstacles to obtaining treatment for hearing loss and depression. However, large well-designed intervention studies are needed (and some are already underway) to determine whether treatment of depression and hearing loss reduces the risk of downstream cognitive decline.

Another limitation of our findings includes ascertainment of late-life depression using a score threshold from a rating scale rather than a structured diagnostic interview by a mental health professional may be considered another limitation. However, the 15 -item GDS is well-documented to be a valid screening instrument for depressive symptoms, and a score of 5 or greater on this scale has been shown to reliably detect the presence of major depressive episodes diagnosed using structured interviews (27). Offsetting these limitations are numerous strengths of the study, most notably including a large sample size, longitudinal follow-up of a carefully constructed cohort, high-quality cognitive assessments, and rigorous classification of cognitive status.

\section{Conclusions}

Data from this study suggest that individuals with ARHL and depression identify a clinical phenotype at risk for dementia. Age-related hearing loss, dementia, and depression both individually and collectively, are responsible for tremendous public health costs, including extensive disability, morbidity, and mortality. Further understanding the mechanisms linking these disorders of later life may facilitate identification and dissemination of interventions (including both hearing remediation and antidepressant medications) capable of protecting brain health during aging and altering the clinical trajectories of at-risk individuals.

\section{Funding}

There were no funding sources for the coauthors of the manuscript. However, the National Alzheimer's Coordinating Center's database is funded by NIA/NIH Grant U01 AG016976. NACC data are contributed by the NIA-funded ADCs: P30 AG019610 (PI Eric Reiman, MD), P30 AG013846 (PI Neil Kowall, MD), P30 AG062428-01 (PI James Leverenz, MD), P50 AG008702 (PI Scott Small, MD), P50 AG025688 (PI Allan Levey, MD, PhD), P50 AG047266 (PI Todd Golde, MD, PhD), P30 AG010133 (PI Andrew Saykin, PsyD), P50 AG005146 (PI Marilyn Albert, PhD), P30 AG062421-01 (PI Bradley Hyman, MD, PhD), P30 AG062422-01 (PI Ronald Petersen, MD, PhD), P50 AG005138 (PI Mary Sano, PhD), P30 AG008051 (PI Thomas Wisniewski, MD), P30 AG013854 (PI Robert 
Vassar, PhD), P30 AG008017 (PI Jeffrey Kaye, MD), P30 AG010161 (PI David Bennett, MD), P50 AG047366 (PI Victor Henderson, MD, MS), P30 AG010129 (PI Charles DeCarli, MD), P50 AG016573 (PI Frank LaFerla, PhD), P30 AG062429-01 (PI James Brewer, MD, PhD), P50 AG023501 (PI Bruce Miller, MD), P30 AG035982 (PI Russell Swerdlow, MD), P30 AG028383 (PI Linda Van Eldik, PhD), P30 AG053760 (PI Henry Paulson, MD, PhD), P30 AG010124 (PI John Trojanowski, MD, PhD), P50 AG005133 (PI Oscar Lopez, MD), P50 AG005142 (PI Helena Chui, MD), P30 AG012300 (PI Roger Rosenberg, MD), P30 AG049638 (PI Suzanne Craft, PhD), P50 AG005136 (PI Thomas Grabowski, MD), P30 AG06271501 (PI Sanjay Asthana, MD, FRCP), P50 AG005681 (PI John Morris, MD), P50 AG047270 (PI Stephen Strittmatter, MD, PhD).

\section{Conflict of Interest}

Drs. Brewster, Rutherford, Brown, Zilcha-Mano, Wall, Roose, Hu, as well as Ms. Stein have no conflicts of interest of financial disclosures to report. Dr. Golub received travel expenses for industry-sponsored meetings (Cochlear, Advanced Bionics, Oticon Medical), consulting fees or honoraria (Oticon Medical, Auditory Insight, Optinose, Abbott, Decibel Therapeutics), and the Department of Otolaryngology received unrestricted educational grants (Storz, Stryker, Acclarent, 3NT, Decibel Therapeutics).

\section{Acknowledgments}

We would like to certify that this manuscript represents valid work and that neither this manuscript nor one with substantially similar content under our authorship has been published or is being considered for publication elsewhere.

\section{Author Contributions}

Drs. Rutherford and Brewster have had full access to the data analyzed here as well as complete freedom to direct its reporting. Each author (Drs. Brewster, Zilcha-Mano, Wall, Brown, Roose, Golub, Rutherford, Hu, and Ms. Stein) in the manuscript contributed to the literature search, study design, data interpretation, and writing of the manuscript. Drs. Brewster, Zilcha-Mano, Rutherford, Brown, Wall, and Hu contributed to the data analysis and figures presented.

\section{References}

1. Collins JG. Prevalence of selected chronic conditions: United States 1990-1992. Vital Health Stat 10 1997;194:1-89. doi:10.2105/ AJPH.2016.303299

2. Goman AM, Lin FR. Prevalence of hearing loss by severity in the United States. Am J Public Health. 2016;106(10):1820-1822. doi:10.2105/ AJPH.2016.303299

3. Cooper JC, Gates GA. Hearing in the elderly-the Framingham cohort, 1983-1985: part II. Prevalence of central auditory processing disorders. Ear Hear. 1991;12(5):304-311. doi:10.1097/00003446-199110000-00002

4. Reed NS, Altan A, Deal JA, et al. Trends in health care costs and utilization associated with untreated hearing loss over 10 years. JAMA Otolaryngol Head Neck Surg. 2019;145(1):27-34. doi:10.1001/jamaoto.2018.2875

5. Kamil RJ, Betz J, Powers BB, et al; Health ABC Study. Association of hearing impairment with incident frailty and falls in older adults. J Aging Health. 2016;28(4):644-660. doi:10.1177/0898264315608730

6. Global Burden of Disease Study 2013 Collaborators. Global, regional, and national incidence, prevalence, and years lived with disability for 301 acute and chronic diseases and injuries in 188 countries, 1990-2013: a systematic analysis for the Global Burden of Disease Study 2013. Lancet 2015;386(9995):743-800. doi:10.1016/S0140-6736(15)60692-4

7. Deal JA, Betz J, Yaffe K, et al; Health ABC Study Group. Hearing impairment and incident dementia and cognitive decline in older adults: the Health ABC Study. J Gerontol A Biol Sci Med Sci. 2017;72:703-709. doi:10.1093/gerona/glw069

8. Lin FR, Metter EJ, O'Brien RJ, Resnick SM, Zonderman AB, Ferrucci L. Hearing loss and incident dementia. Arch Neurol. 2011;68(2):214-220. doi:10.1001/archneurol.2010.362

9. Panza F, Solfrizzi V, Logroscino G. Age-related hearing impairment-a risk factor and frailty marker for dementia and AD. Nat Rev Neurol. 2015;11(3):166-175. doi:10.1038/nrneurol.2015.12

10. Taljaard DS, Olaithe M, Brennan-Jones CG, Eikelboom RH, Bucks RS. The relationship between hearing impairment and cognitive function: a metaanalysis in adults. Clin Otolaryngol. 2016;41(6):718-729. doi:10.1111/ coa. 12607

11. Albers MW, Gilmore GC, Kaye J, et al. At the interface of sensory and motor dysfunctions and Alzheimer's disease. Alzheimers Dement. 2015;11(1):70-98. doi:10.1016/j.jalz.2014.04.514

12. Golub JS, Brewster KK, Brickman AM, et al. Association of audiometric age-related hearing loss with depressive symptoms among Hispanic individuals. JAMA Otolaryngol Head Neck Surg. 2019;145(2):132-139. doi:10.1001/jamaoto.2018.3270

13. Brewster KK, Ciarleglio A, Brown PJ, et al. Age-related hearing loss and its association with depression in later life. Am J Geriatr Psychiatry. 2018;26(7):788-796. doi:10.1016/j.jagp.2018.04.003

14. Rutherford BR, Brewster K, Golub JS, Kim AH, Roose SP. Sensation and psychiatry: linking age-related hearing loss to late-life depression and cognitive decline. Am J Psychiatry. 2018;175(3):215-224. doi:10.1176/appi. ajp.2017.17040423

15. Byers AL, Yaffe K. Depression and risk of developing dementia. Nat Rev Neurol. 2011;7(6):323-331. doi:10.1038/nrneurol.2011.60

16. Brommelhoff JA, Gatz M, Johansson B, McArdle JJ, Fratiglioni L, Pedersen NL. Depression as a risk factor or prodromal feature for dementia? Findings in a population-based sample of Swedish twins. Psychol Aging. 2009;24(2):373-384. doi:10.1037/a0015713

17. Vilalta-Franch J, López-Pousa S, Llinàs-Reglà J, Calvó-Perxas L, MerinoAguado J, Garre-Olmo J. Depression subtypes and 5-year risk of dementia and Alzheimer disease in patients aged 70 years. Int J Geriatr Psychiatry. 2013;28(4):341-350. doi:10.1002/gps.3826

18. Green RC, Cupples LA, Kurz A, et al. Depression as a risk factor for Alzheimer disease: the MIRAGE Study. Arch Neurol. 2003;60(5):753759. doi:10.1001/archneur.60.5.753

19. Ownby RL, Crocco E, Acevedo A, John V, Loewenstein D. Depression and risk for Alzheimer disease: systematic review, meta-analysis, and metaregression analysis. Arch Gen Psychiatry. 2006;63(5):530-538. doi:10.1001/archpsyc.63.5.530

20. Kaup AR, Byers AL, Falvey C, et al. Trajectories of depressive symptoms in older adults and risk of dementia. JAMA Psychiatry. 2016;73(5):525-531. doi:10.1001/jamapsychiatry.2016.0004

21. Mirza SS, Wolters FJ, Swanson SA, et al. 10-year trajectories of depressive symptoms and risk of dementia: a population-based study. Lancet Psychiatry. 2016;3(7):628-635. doi:10.1016/S2215-0366(16)00097-3

22. Sonsin-Diaz N, Gottesman RF, Fracica E, et al. Chronic systemic inflammation is associated with symptoms of late-life depression: the ARIC Study. Am J Geriatr Psychiatry. 2020;28(1):87-98. doi:10.1016/j. jagp.2019.05.011

23. Sheline YI, Disabato BM, Hranilovich J, et al. Treatment course with antidepressant therapy in late-life depression. Am J Psychiatry. 2012;169(11):1185-1193. doi:10.1176/appi.ajp.2012.12010122

24. Manning KJ, Alexopoulos GS, Banerjee S, et al. Executive functioning complaints and escitalopram treatment response in late-life depression. Am J Geriatr Psychiatry. 2015;23(5):440-445. doi:10.1016/j.jagp.2013.11.005

25. Barnes DE, Yaffe K. The projected effect of risk factor reduction on Alzheimer's disease prevalence. Lancet Neurol. 2011;10(9):819-828. doi:10.1016/S1474-4422(11)70072-2

26. Yesavage JA, Brink TL, Rose TL, et al. Development and validation of a geriatric depression screening scale: a preliminary report. J Psychiatr Res. 1982;17(1):37-49. doi:10.1016/0022-3956(82)90033-4 
27. Brown PJ, Woods CM, Storandt M. Model stability of the 15 -item Geriatric Depression Scale across cognitive impairment and severe depression. Psychol Aging. 2007;22(2):372-379. doi:10.1037/0882-7974.22.2.372

28. Morris JC. The Clinical Dementia Rating (CDR): current version and scoring rules. Neurology. 1993;43:2412-2414. doi:10.1212/ wnl.43.11.2412-a

29. Kaufer DI, Cummings JL, Ketchel P, et al. Validation of the NPI-Q, a brief clinical form of the Neuropsychiatric Inventory. J Neuropsychiatry Clin Neurosci. 2000;12:233-239. doi:10.1176/jnp.12.2.233

30. Pfeffer RI, Kurosaki TT, Harrah CH Jr, Chance JM, Filos S. Measurement of functional activities of older adults in the community. J Gerontol. 1982;37:323-329. doi:10.1093/geronj/37.3.323

31. Weintraub S, Salmon D, Mercaldo N, et al. The Alzheimer's Disease Centers' Uniform Data Set (UDS): the neuropsychologic test battery. Alzheimer Dis Assoc Disord. 2009;23(2):91-101. doi:10.1097/ WAD.0b013e318191c7dd

32. Beekly DL, Ramos EM, Lee WW, et al; NIA Alzheimer's Disease Centers. The National Alzheimer's Coordinating Center (NACC) database: the Uniform Data Set. Alzheimer Dis Assoc Disord. 2007;21(3):249-258. doi:10.1097/WAD.0b013e318142774e

33. VanderWeele TJ, Vansteelandt S. Mediation analysis with multiple mediators. Epidemiol Methods. 2014;2(1):95-115. doi:10.1515/em-2012-0010

34. Golub JS, Lin FR, Lustig LR, Lalwani AK. Prevalence of adult unilateral hearing loss and hearing aid use in the United States. Laryngoscope. 2018;128(7):1681-1686. doi:10.1002/lary.27017

35. Chien W, Lin FR. Prevalence of hearing aid use among older adults in the United States. Arch Intern Med. 2012;172(3):292-293. doi:10.1001/ archinternmed.2011.1408

36. Gallagher D, Kiss A, Lanctot K, Herrmann N. Depression and risk of alzheimer dementia: a longitudinal analysis to determine predictors of increased risk among older adults with depression. Am J Geriatr Psychiatry. 2018;26(8):819-827. doi:10.1016/j.jagp.2018.05.002

37. Gallagher D, Kiss A, Lanctot KL, Herrmann N. Toward prevention of mild cognitive impairment in older adults with depression: an observational study of potentially modifiable risk factors. J Clin Psychiatry. 2018:80(1):18m12331. doi:10.4088/JCP.18m12331

38. Steenland K, Karnes C, Seals R, Carnevale C, Hermida A, Levey A. Late-life depression as a risk factor for mild cognitive impairment or Alzheimer's disease in 30 US Alzheimer's disease centers. J Alzheimers Dis. 2012;31(2):265-275. doi:10.3233/JAD-2012-111922

39. Zeki Al Hazzouri A, Vittinghoff E, Byers A, et al. Long-term cumulative depressive symptom burden and risk of cognitive decline and dementia among very old women. J Gerontol A Biol Sci Med Sci. 2014;69(5):595601. doi:10.1093/gerona/glt139

40. Mick P, Kawachi I, Lin FR. The association between hearing loss and social isolation in older adults. Otolaryngol Head Neck Surg. 2014;150(3):378384. doi:10.1177/0194599813518021

41. Wild CJ, Yusuf A, Wilson DE, Peelle JE, Davis MH, Johnsrude IS. Effortful listening: the processing of degraded speech depends critically on attention. J Neurosci. 2012;32(40):14010-14021. doi:10.1523/ JNEUROSCI.1528-12.2012

42. Lin FR, Ferrucci L, An Y, et al. Association of hearing impairment with brain volume changes in older adults. Neuroimage. 2014;90:84-92. doi:10.1016/j.neuroimage.2013.12.059

43. Sindhusake D, Mitchell P, Smith W, et al. Validation of self-reported hearing loss. The Blue Mountains Hearing Study. Int J Epidemiol. 2001;30(6):1371-1378. doi:10.1093/ije/30.6.1371

44. Nondahl DM, Cruickshanks KJ, Wiley TL, Tweed TS, Klein R, Klein BE. Accuracy of self-reported hearing loss. Audiology. 1998;37(5):295-301. doi:10.3109/00206099809072983 